Z Gerontol Geriat 2010 • 43:5-7 DOI 10.1007/s00391-009-0093-x

Online publiziert: 21. Januar 2010

(c) Springer-Verlag 2010

\author{
R.D. Hirsch ${ }^{1}$. W. Ruch ${ }^{2}$ \\ ${ }^{1}$ Abteilung für Gerontopsychiatrie und -psychotherapie, LVR-Klinik Bonn \\ 2 Fachrichtung für Persönlichkeitspsychologie und Diagnostik, \\ Psychologisches Institut, Universität Zürich
}

\title{
Heiterkeit und Humor im Alter
}

\section{Ergebnisse aktueller Studien}

„Humor zu analysieren ist wie das Sezieren eines Frosches - niemand tut es gern und am Ende ist der Gegenstand der Analyse tot" (unbekannter Autor). So könnte man die Gefahr beschreiben, die besteht, wenn Wissenschaftler sich mit dem Thema „Humor" befassen. Und dennoch versuchen wir, uns diesem Themenbereich zu nähern und wissenschaftliche Untersuchungen und Folgerungen vorzustellen, ohne dabei den Humor austrocknen zu wollen. Wir möchten den Blick auf eine Fähigkeit und Kompetenz richten, die in der positiven Psychologie als Charakterstärke beschrieben wird. Über diese verfügt jeder Mensch mehr oder weniger. Bekanntlich „hat man ihn“, den „Humor“. Humor gilt als eine Eigenschaft, auf deren Besitz jeder Mensch Wert legt [3]. Humorlos möchte keiner sein. Humor hat jeder! Davon ist man überzeugt! „Sie haben keinen Humor" wird meist als Beleidigung aufgefasst.

Humor in der Medizin und Pflege hat sich dagegen anscheinend noch nicht durchgesetzt. Nicht umsonst spricht man vom „Patienten“ (Leidenden) und nicht vom Menschen, der eine Erkrankung hat. Allerdings bezeichnet Dührssen [2] den Humor, „den wir für unsere Behandlungen so dringend benötigen und der es verhindert, dass schwierige, aber nicht unlösbare Situationen eines Kranken zum Melodrama werden“, als erste Persönlichkeitskonstante, über die nicht nur ein Psychoanalytiker verfügen sollte.

Leider existieren über den Humor vielfältige Mythen, Vorurteile, Volks- weisheiten oder als „erwiesen“ bezeichnete Aussagen, deren Belegbarkeit noch offen ist - insbesondere in der deutschsprachigen Literatur. So heißt es z. B., dass Kinder am Tag 40o-mal lachen und Erwachsene höchstens 20-mal. Auch wenn diese Behauptung immer wieder zitiert wird, gibt es hierzu keine empirische Untersuchung. Auch im Rahmen der Therapie finden sich zwar vielfältige Untersuchungen und Beschreibungen, welche die Wirksamkeit von Humor in der Therapie belegen sollen, doch ist deren wissenschaftliche Aussagefähigkeit bis heute sehr lückenhaft. Leider ist Martin [5] immer noch zuzustimmen, dass Masse allein noch nicht Klasse bedeutet und die ungelösten Probleme der Grundlagenforschung sich in den angewandten Studien fortsetzen.

Wird Humor auch oft mit Lachen gleichgesetzt, so muss Lachen kein Zeichen von Humor sein. Auch beruht die Mehrzahl der Witze, über die wir lachen, auf Beschämung, Erniedrigung und Kleinmachen. Sie als humorvoll zu bezeichnen, verwischt die kreative lebensfördernde Macht des Humors. So schreibt Nietzsche [9]: „Nicht durch Zorn, sondern durch Lachen tötet man. "Die auf den deutschen Schriftsteller Otto Julius Bierbaum (18651910) zurückgehende Redeweise „Humor ist, wenn man trotzdem lacht" gilt heute als Volksweisheit, auch wenn hiermit zwar die „Trotzmacht Humor“ pointiert wiedergegeben ist, die Verbindung zum Lachen aber eher unzutreffend ist. 
Die wissenschaftliche Erforschung des Humors ist in den letzten Jahrzehnten vor allem international weit vorangeschritten. Forschungsergebnisse werden in verschiedenen disziplinären Zeitschriften veröffentlicht und seit mehr als 20 Jahren existiert die interdisziplinäre, auf das Thema spezialisierte Zeitschrift HUMOR: International Journal of $\mathrm{Hu}$ mor Research. Es gibt jährlich einen Kongress (s. http://www.humorstudies.org), auf dem Forschungsergebnisse vorgestellt werden, und auch eine „summer school“ (www.humoursummerschool.org), die in das Thema einführt. Der Stand der Forschung in vielen Bereichen wurde kürzlich in einem informativen Einführungswerk zusammengefasst [10]. International hat sich die Humorforschung also etabliert. Das Aufkommen der positiven Psychologie, die bekanntlich das "gute Leben" besser erforschen möchte, wird die Untersuchung des Humors noch weiter verstärken. Sie sieht Humor als eine von 24 Charakterstärken, die das gute Leben begünstigen.

Hingegen sind wissenschaftliche Aussagen, inwieweit Humor im Alter eine Rolle spielt, derzeit noch rar. Hinweise hierfür geben Ruch u. Zweyer [11]. Sie beobachteten, dass Messwerte zur „, heiteren Gelassenheit" bei über 6o-Jährigen deutlich höher liegen als bei Jüngeren. Hirsch u. Kranzhoff [4] folgerten aufgrund einer Befragung von älteren Menschen, die in einer gerontopsychiatrischen Klinik behandelt wurden, dass der Stellenwert von Humor für sie hoch ist und auch ältere Menschen noch Humor lernen können. Bowling [1] diskutiert, dass Humor als ein Bestandteil des erfolgreichen Alterns gesehen werden soll. Humor nimmt damit eine prominente Stellung unter einem Duzend weiterer Kriterien ein. Die Autorin lässt sich allerdings wenig darauf ein, was denn genau unter „Humor" verstanden werden soll, wie man ihn misst, was seine Ursachen und Konsequenzen sind, wie man ihn fördert etc. Die Lebensspannenforschung hat das Thema "Humor" mit dem Buch von Nahemov et al. [8] sehr früh aufgegriffen, aber zu einer Zeit, in der unter Humor noch primär das Verstehen und Produzieren von Witzen etc. verstanden wurde. Was ist mit den anderen Kom- ponenten des Humors? Welche Rolle spielen sie im Leben älterer Personen?

Das vorliegende Heft will dazu beitragen, diese Lücke zu verringern. Ziel ist es, mit Originalbeiträgen und Übersichtsarbeiten verschiedene Aspekte von Humor, Lachen und Heiterkeit im Alter genauer zu beleuchten. Im ersten Beitrag (Ruch, Proyer, Weber) wird ein kurzer Überblick über die positive Psychologie gegeben und Humor als eine Charakterstärke definiert. Es wird darauf eingegangen, inwiefern Humor das Altern positiv beeinflusst und das Leben lebenswert macht. Möglichkeiten der Messung von Humor werden ebenso diskutiert wie deren Auswirkungen für die Forschung und Praxis.

Es folgen empirische Ergebnisse einer großen Online-Untersuchung, die auf einem Fragebogen für Humor als Charakterstärke sowie einer Skala zur Lebenszufriedenheit und zum Glück basiert (Ruch, Proyer, Weber). Insgesamt bearbeiteten ca. 43.00o Probanden diese Fragebögen. Es zeigte sich, dass die Humorwerte bis zum Alter von 50 Jahren sinken, dass aber 61- bis 65-jährige Männer deutlich höhere Werte aufweisen. Ein weiteres Ergebnis ist, dass Humor auch bei den älteren Probanden mit Lebenszufriedenheit sowie mit „vergnüglichem“ und „engagiertem“, am wenigsten aber mit dem „bedeutsamen“ Leben korreliert.

Ein weiterer Beitrag (Proyer, Ruch, Müller) vergleicht den Sinn für Humor, basierend auf dem theoretischen Modell von McGhee [7], in verschiedenen Altersgruppen $(n=979)$. Ein Ergebnis ist, dass eine spielerische Haltung und der Sinn für Humor über die Altersgruppen hinweg relativ stabil sind. Allerdings zeigen sich für die ältesten Teilnehmerinnen die höchsten Werte in positiver Stimmung. Diese geben an, dass sie weniger und weniger leicht lachen als jüngere Teilnehmerinnen. Interessant ist auch der Befund, dass ältere Teilnehmer, die sich häufiger mit Freunden treffen, auch höhere Werte in verschiedenen Aspekten des Sinns für Humor erzielen als diejenigen, die sich weniger häufig mit Freunden treffen.

In einem Übersichtsreferat wird der Entwicklung von Lachen und Humor in den verschiedenen Lebensphasen nachgegangen (Falkenberg). Das Humorverständnis, die Humorproduktion und die
Funktion von Humor ändern sich im Laufe des Lebens. Sie sind wesentlich durch kognitive, sprachliche und soziale Fähigkeiten, die für die einzelnen Lebensabschnitte dargestellt werden, bestimmt. Bei älteren Menschen fällt auf, dass für sie der Humor als Bewältigungsmechanismus von Stress eine besondere Bedeutung hat.

Der Stellenwert der Neurobiologie und deren Aussagen über psychische Phänomene haben in den letzten Jahren erheblich zugenommen. So werden seit einiger Zeit Untersuchungen zur Verarbeitung von witzigen Stimuli im Gehirn durchgeführt (Wild). Dargestellt werden Ergebnisse zu den an Witzverarbeitung beteiligten Strukturen sowie neuropsychologische Untersuchungen zu den notwendigen kognitiven Werkzeugen wie z. B. das Arbeitsgedächtnis und die mentale Flexibilität. Ein Ergebnis ist auch, dass Humor eine komplexe Fähigkeit ist, die viele Gehirngebiete involviert. Die intellektuelle Reaktion auf Witze scheint durch Alterns-prozesse verlangsamt, Humor als Ganzes aber eher „alterungsresistent“ $\mathrm{zu}$ sein.

Einen Überblick über die aktuelle Literatur zur Gelotophobie (der Angst vor dem Ausgelachtwerden) mit dem Hauptaugenmerk auf altersspezifische Aspekte gibt der Artikel von Platt, Ruch und Proyer. Konstant ist der Befund, dass Menschen mit Gelotophobie dazu neigen, positiv motiviertes Lächeln und Lachen fehlerhaft zu interpretieren. Vorgestellt werden neue Ergebnisse, die zeigen, dass altersspezifische Vulnerabilitäten zusätzliche Quellen für das Ausgelachtwerden sein können. Die Gelotophobie scheint für ältere Menschen ein besonderes Problem darzustellen.

Für die Praxis ist die Anwendung von Humor als Gruppenpsychotherapie für alte Menschen mit einer Depression von besonderer Bedeutung (Hirsch, Junglas, Konradt, Jonitz). Anhand von verschiedenen Fragebögen und Testverfahren wurden alte Menschen mit einer Depression im stationären Setting untersucht. Die Experimentalgruppe nahm kontinuierlich an einer zweimal wöchentlich stattfindenden Humorgruppe teil, die Kontrollgruppe nicht. Im Vergleich beider Gruppen lassen sich eine Vielzahl von Einzelaspekten aufzeigen. So stiegen bei 
den Teilnehmern der Humorgruppe die Werte für die Resilienz und Lebenszufriedenheit signifikant an. Bei Patienten mit mittelschwerer und schwerer Depression (definiert durch die geriatrische Depressionsskala, GDS) wurden außerdem signifikante Verbesserungen bezüglich des $\mathrm{Zu}$ standes (State) Heiterkeit, Ernsthaftigkeit, schlechte Laune sowie Lebenszufriedenheit beobachtet. Die Studie belegt die Effektivität der Humorgruppe als spezifische Form der Gruppenpsychotherapie.

Clowns galten schon immer als Gesundheitsförderer. In einem Übersichtsartikel werden Berichte über und von Clowns, meist Klinikclowns, auch „Gericlowns" genannt, dargestellt, und es wird auf die wenigen Untersuchungen, die es hierzu gibt, eingegangen (Rösner). Klinikclowns sind in der Gerontopsychiatrie und im Altenpflegeheim zwar noch selten anzutreffen, allerdings ist deren positive Wirkung grundsätzlich kaum noch zu bestreiten. Beispielsweise sind Clowns imstande, zu Menschen mit Demenz einen Zugang finden. Sie können dazu beitragen, deren Wohlbefinden zu steigern, herausfordernde Verhaltensweisen zu verringern und damit auch die Lebensqualität zu verbessern. Zudem wird das Arbeitsklima des Pflegepersonals positiv beeinflusst und eine kreative Umgangsweise mit schwierigen Situationen gefördert.

Die vorgestellten Artikel verdeutlichen, dass Heiterkeit und Humor für alte Menschen wichtige Aspekte sind und deren Förderung die Lebensqualität positiv beeinflussen kann. Alte Menschen verfügen trotz inter- und intraindividueller Schwankungsbreite ihrer Fähigkeiten sehr wohl noch über Humor. Deutlich wird, dass nicht nur in der Grundlagenforschung, sondern auch in der Anwendung von Humor in der Therapie und Pflege noch viele Fragen offen sind und der weiteren Erforschung bedürfen. Andererseits ist aber festzustellen, dass ein "gelotologischer Nihilismus" keineswegs angebracht ist. Leider gilt noch immer die Bemerkung von Lorenz [6]: „..., aber ich glaube,... daß wir heute den Humor noch nicht ernst genug nehmen. "Der Beobachtung von Sradj u. Sradj [12] mag zwar die wissenschaftliche Evidenz noch fehlen, doch trifft der Praktiker sie immer wieder an: „Ältere Menschen entdecken in der Regel - vielleicht durch das Bewusstwerden des ihnen Entgangenen - Nutzen und Notwendigkeit des Humors wieder, agieren bewusster und gehen das ,Abenteuer' des Humors häufiger ein“.

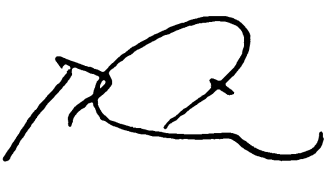

R.D. Hirsch

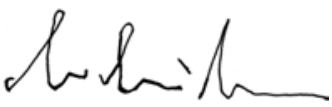

W. Ruch

\section{Korrespondenzadresse}

Prof. Dr. Dr. R.D. Hirsch

Abteilung für Gerontopsychiatrie und -psychotherapie, LVR-Klinik Bonn, Kaiser-Karl-Ring 20, 53111 Bonn

r.hirsch@lvr.de

\section{Prof. Dr. W. Ruch}

Fachrichtung für Persönlichkeitspsychologie und Diagnostik, Psychologisches Institut, Universität Zürich, Binzmühlestr. 14/7, 8050 Zürich,

Schweiz

w.ruch@psychologie.uzh.ch

\section{Literatur}

1. Bowling A (2007) Aspirations for older age in the 21st century: what is successful aging? Int J Aging Hum Dev 64 (3):263-297

2. Dührssen A (1972) Analytische Psychotherapie in Theorie, Praxis und Ergebnisse. Vandenhoeck \& Ruprecht, Göttingen, S 297

3. Hirsch E (1991) Der Witzableiter. dtv, München

4. Hirsch RD, Kranzhoff EU (2004) Humorgruppe mit alten Menschen: Ergebnisse einer therapiebegleitenden Untersuchung. Gruppenpsychother Gruppendynamik 40:106-129

5. Martin RA (2000) Humor, laughter, and physical health: methodological issues and research findings. Psychological Bulletin 127(4):504-519

6. Lorenz K (1993) Das sogenannte Böse. dtv, München, $\mathrm{S} 257$

7. McGhee $P$ (1996) Health, healing and the amuse system: humor as survival training. Kendall/Hunt, Dubuque/lQ

8. Nahemov L, McCluskey-Fawcett KA, McGhee PE (1986) (eds) Humor and aging. Academic Press, New York

9. Nietzsche F (1975) Also sprach Zarathustra. Körner, Stuttgart

10. Raskin V (2008) (ed) A primer of humor. Mouton de Gruyter, Berlin
11. Ruch W, Zweyer K (2001) Ergebnisse der Forschung zu Humor und Heiterkeit. In: Hirsch RD, Bruder J, Radebold $\mathrm{H}$ (Hrsg) Heiterkeit und Humor im Alter. Schriftenreihe der Deutschen Gesellschaft für Gerontopsychiatrie und -psychotherapie, Bd 2. Kohlhammer, Stuttgart, S 9-43

12. Sradj M, Sradj N (1988) Das Abenteuer des Humors. Dt Ärztebl 85:B-514 